\title{
Computing Higher Dimensional Digital Homotopy Groups
}

\author{
Elif Tugce Meric ${ }^{1}$, Tane Vergili ${ }^{2}$ and Ismet Karaca ${ }^{2, *}$ \\ ${ }^{1}$ Department of Mathematics, Faculty of Science and Arts, Celal Bayar University, 45140 Manisa, Turkey \\ ${ }^{2}$ Department of Mathematics, Faculty of Science, Ege University, 35100 Bornova, Izmir, Turkey
}

Received: 9 Sep. 2013, Revised: 7 Dec. 2013, Accepted: 8 Dec. 2013

Published online: 1 Sep. 2014

\begin{abstract}
In this paper, we study out a method for computing digital homotopy groups in higher dimensions. We investigate the relation between a digital image and its $n^{\text {th }}$ homotopy group when $n$ is greater than 1 and show that a digital covering map which is a radius 2 local isomorphism induces an isomorphism between digital homotopy groups in higher dimensions.
\end{abstract}

Keywords: digital homotopy groups, digital covering map, radius 2 local isomorphism

\section{Introduction}

Digital topology has an important role in computer vision, image processing and computer graphics which are useful in many other areas. It investigates the properties of digital images on $\mathbb{Z}^{n}$ by using methods of algebraic topology. It was introduced by Rosenfeld in $20^{\text {th }}$ century. His works on the subject played an important role in establishing and developing the field. After Rosenfelds works, this area has been studied by many of researchers (Kong, Kopperman, Kovalevsky, Malgouyres, Ayala, Boxer, Chen, Han, Karaca and others).

Digital fundamental groups help to classify digital images as in algebraic topology. This notion was introduced by Kong [14]. Kong's construction wasn't parallel to the classical construction of the fundamental group of a topological space. So, Boxer [2] has given a classical construction in calculating the fundamental groups of digital images by using the notion of digital homotopy introduced in [1]. After digital fundamental group was defined, new methods were devised for computing it.

The digital covering space is one of the tools for computing the digital fundamental groups. Han [9] introduces the digital covering space and digital lifting notions, computes a digital homotopy group of some digital image. The theory of digital covering space has been developed by Boxer and Karaca [4,7,8,9] by deriving digital analogs of classical results of algebraic topology. Boxer [4] has discussed a digital version of the universal covering space and Boxer and Karaca [7] have classified the digital covering space by the conjugacy class corresponding a digital covering space.

Karaca and Vergili [12] have explored the digital relative homotopy relation between continuous functions whose domains are $n$-cubes and which map the boundary of an $n$-cube to a fixed point. They have introduced n-th homotopy groups of pointed digital images via this relation and obtained some results which are valid for topological spaces.

In higher dimensions, the digital homotopy groups are sometimes very complicated despite their simple definitions. However, if the digital image has a digital covering, then in this case there is a certain relation between their higher homotopy groups. After Han [10] has presented radius 2 local isomorphism, Boxer [4] has showed that a digital covering map which is a radius 2 local isomorphism induces a monomorphism between digital fundamental groups. The main goal of this paper is to investigate an analogus result from algebraic topology and introduce a new method for computation of digital homotopy groups by using covering spaces. We show that the higher dimensional digital homotopy group of a

\footnotetext{
${ }^{*}$ Corresponding author e-mail: ismet.karaca@ege.edu.tr
} 
digital image is isomorphic to the higher dimensional digital homotopy group of its covering space in each dimension.

This paper is organised as follows. Some basic knowledge is provided in Section 2. In the next section, we recall the homotopy group construction which is given by Boxer [2], Karaca and Vergili [12] and recall some properties of these groups. In Section 4, we investigate the relation between a digital covering space and higher dimensional digital homotopy group of a digital image. We obtain that a covering map induces isomorphism between homotopy groups of pointed digital images in higher dimensions when it is a radius 2 local isomorphism. In the last section, we get some conclusions.

\section{Preliminaries}

Let $\mathbb{Z}$ represent the set of integers. A (binary) digital image is a pair $(X, \kappa)$, where $X$ is a subset of $\mathbb{Z}^{n}$ for some positive integer $n$ and $\kappa$ indicates some adjacency relations on $X$. There are $n$ adjacency relation for $\mathbb{Z}^{n}$ to be used in the study of digital images. The following terminology is used in [14]. Two points $p$ and $q$ in $\mathbb{Z}^{2}$ are 8 -adjacent if they are distinct and differ by at most 1 in each coordinate; $p$ and $q$ in $\mathbb{Z}^{2}$ are 4-adjacent if they are 8 -adjacent and differ in exactly one coordinate. Two points $p$ and $q$ in $\mathbb{Z}^{3}$ are 26-adjacent if they are distinct and differ by at most 1 in each coordinate; they are 18 -adjacent if they are 26-adjacent and differ in at most two coordinates; they are 6-adjacent if they are 18 -adjacent and differ in exactly one coordinate. The adjacencies are generalized as follows [6]. Let $l, n$ be positive integers, $1 \leq l \leq n$ and consider two distinct points $p=\left(p_{1}, p_{2}, \ldots, p_{n}\right), q=\left(q_{1}, q_{2}, \ldots, q_{n}\right) \in \mathbb{Z}^{n}, p$ and $q$ are $\kappa_{l}$-adjacent if there are at most $l$ distinct coordinates $j$ for which $\left|p_{j}-q_{j}\right|=1$, and for all other coordinates $j, p_{j}=q_{j}$. A $\kappa_{l}$-adjacency relation on $\mathbb{Z}^{n}$ may be denoted by the number of points that are adjacent to a point $p \in \mathbb{Z}^{n}$. For example, $\kappa_{1}$-adjacent points of $\mathbb{Z}^{2}$ are called 4-adjacent; $\kappa_{2}$-adjacent points of $\mathbb{Z}^{2}$ are called 8 -adjacent; and in $\mathbb{Z}^{3}, \kappa_{1^{-}}, \kappa_{2}$, and $\kappa_{3}$-adjacent points are called 6-adjacent, 18-adjacent, and 26-adjacent, respectively.

For $a, b \in \mathbb{Z}$ with $a \leq b$, the set

$$
[a, b]_{\mathbb{Z}}=\{z \in \mathbb{Z}: a \leq z \leq b\}
$$

is called a digital interval [2] in which 2-adjacency is assumed.

Let $(X, \kappa)$ be a digital image. A $\kappa$-path [15] from $x$ to $y$ in $X$ is a sequence $\left(x=x_{0}, x_{1}, \ldots, x_{m-1}, x_{m}=y\right)$ in $X$ such that each point $x_{i}$ is $\kappa$-adjacent to $x_{i+1}$ for $i \in[0, m-1]_{\mathbb{Z}}$. The natural number $m$ is called length of the path [2],[15].
If $x_{0}=x_{m}$, then tke $\kappa$-path is said to be closed. Two distinct points $x, y \in X$ are $\kappa$-connected if there is a $\kappa$-path from $x$ to $y$ in $X$ and if any two points in $X$ are $\kappa$-connected, then $X$ is called $\kappa$-connected [15]. A $\kappa$-component of a digital image $X$ is a maximal $\kappa$-connected subset of $X$. The $\kappa$ neighborhood [9] of $x_{0} \in X$ with radius $\varepsilon$ is the set

$$
N_{\kappa}\left(x_{0}, \varepsilon\right)=\left\{x \in X \mid l_{\kappa}\left(x_{0}, x\right) \leq \varepsilon\right\},
$$

where $l_{\kappa}\left(x_{0}, x\right)$ is the length of a shortest $\kappa$-path from $x_{0}$ to $x$.

Let $X \subset \mathbb{Z}^{n_{0}}$ and $Y \subset \mathbb{Z}^{n_{1}}$. Let $\kappa_{i}$ be an adjacency relation defined on $\mathbb{Z}^{n_{i}}, i \in\{0,1\}$. We say that a function $f: X \longrightarrow Y$ is $\left(\kappa_{0}, \kappa_{1}\right)$-continuous $[2,4]$ if the image under $f$ of every $\kappa_{0}$-connected subset of $X$ is $\kappa_{1}$-connected subset of $Y$.

The following Proposition is a characterization of $\left(\kappa_{0}, \kappa_{1}\right)$-continuity.

Proposition 2.1. [16,2] Let $X \subset \mathbb{Z}^{n_{0}}$ and $Y \subset \mathbb{Z}^{n_{1}}$ be digital images with $\kappa_{0}$-adjacency and $\kappa_{1}$-adjacency respectively. Then the function $f: X \rightarrow Y$ is $\left(\kappa_{0}, \kappa_{1}\right)$-continuous if and only if for every pair of $\kappa_{0}$-adjacent points $\left\{x_{0}, x_{1}\right\}$ of $X$, either $f\left(x_{0}\right)=f\left(x_{1}\right)$ or $f\left(x_{0}\right)$ and $f\left(x_{1}\right)$ are $\kappa_{1}$-adjacent in $Y$.

Composition preserves digital continuity [2], i.e., if $f: X \longrightarrow Y$ and $g: Y \longrightarrow Z$ are, respectively, $\left(\kappa_{1}, \kappa_{2}\right)$-continuous and $\left(\kappa_{2}, \kappa_{3}\right)$-continuous functions, then the composite function $(g \circ f): X \longrightarrow Z$ is $\left(\kappa_{1}, \kappa_{3}\right)$-continuous.

If $(X, \kappa)$ is a digital image and $A \subset X$, then we call $(\mathrm{X}, \mathrm{A})$ a digital image pair with $\kappa$-adjacency. For digital image pairs $(X, A)$ and $(Y, B)$ with $\kappa_{0}$-adjacency and $\kappa_{1}$-adjacency respectively, a function $f:(X, A) \rightarrow(Y, B)$ is a $\left(\kappa_{0}, \kappa_{1}\right)$-continuous map of digital pairs if $f$ is $\left(\kappa_{0}, \kappa_{1}\right)$-continuous and $f(A) \subset B$. When $A=\{a\}$ and $B=\{b\}$, we write $(X, A)=(X, a),(Y, B)=(Y, b)$ and we say $f$ is a pointed $\left(\kappa_{0}, \kappa_{1}\right)$-continuous map [2] between pointed digital images $(A, a)$ and $(Y, b)$.

Let $\left(X, \kappa_{0}\right)$ and $\left(Y, \kappa_{1}\right)$ be digital images. A function $f: X \rightarrow Y$ is a $\left(\kappa_{0}, \kappa_{1}\right)$-isomorphism [1] if $f$ is $\left(\kappa_{0}, \kappa_{1}\right)$-continuous and bijective and further $f^{-1}: Y \rightarrow X$ is $\left(\kappa_{1}, \kappa_{0}\right)$-continuous.

Definition 2.2. ([2]; see also [13]) Let $X$ and $Y$ be digital images. Let $f, g: X \longrightarrow Y$ be $\left(\kappa_{1}, \kappa_{2}\right)$-continuous functions. Suppose there is a positive integer $m$ and a function

$$
F: X \times[0, m]_{\mathbb{Z}} \rightarrow Y
$$

such

- for all $x \in X, F(x, 0)=f(x)$ and $F(x, m)=g(x)$;

- for all $x \in X$, the induced function $F_{x}:[0, m]_{\mathbb{Z}} \rightarrow Y$ 
defined by $F_{x}(t)=F(x, t)$ for all $t \in[0, m]_{\mathbb{Z}}$ is $\left(2, \kappa_{2}\right)$-continuous; and

- for all $t \in[0, m]_{\mathbb{Z}}$, the induced function $F_{t}: X \rightarrow Y$ defined by $F_{t}(x)=F(x, t)$ for all $x \in X$ is $\left(\kappa_{1}, \kappa_{2}\right)$-continuous.

Then $F$ is a digital $\left(\kappa_{1}, \kappa_{2}\right)$-homotopy between $f$ and $g$, and $f$ and $g$ are digitally $\left(\kappa_{1}, \kappa_{2}\right)$-homotopic in $Y$, and denoted by $f \simeq_{\kappa_{1}, \kappa_{2}} Y$.

Boxer [2] shows that digital $\left(\kappa_{1}, \kappa_{2}\right)$-homotopy is an equivalence relation among digitally continuous functions $f:\left(X, \kappa_{1}\right) \longrightarrow\left(Y, \kappa_{2}\right)$.

Let $A \subset X$ and $f, g: X \rightarrow Y$ be $\left(\kappa_{0}, \kappa_{1}\right)$-continuous functions. A digital homotopy

$$
H: X \times[0, m]_{\mathbb{Z}} \longrightarrow Y
$$

between $f$ and $g$ is called a digital homotopy relative to $A$ between $f$ and $g$ if for all $a \in A$, and for all $t \in[0, m]_{\mathbb{Z}}$, $H(a, t)=f(a)=g(a)$ (see [11]). Then we say that $f$ and $g$ are $\left(\kappa_{0}, \kappa_{1}\right)$-homotopic relative to $A$ in $Y$. If $A=\left\{x_{0}\right\} \subset X$, then $H$ is called a pointed digital homotopy [2] between $f$ and $g$.

Let $c$ be the constant function for some $x_{0} \in X$ defined by $c(x)=x_{0}$ for all $x \in X$. A digital image $(X, \kappa)$ is said to be $\kappa$-contractible $[2,13]$ if its identity map is $(\kappa, \kappa)$ homotopic to the constant function $c$ for some $x_{0} \in X$. If the homotopy holds $x_{0}$ fixed, we say $\left(X, x_{0}\right)$ is pointed $\kappa$ contractible.

\section{Digital Homotopy Groups}

Homotopy groups are important invariants in algebraic topology. Boxer [3] shows that digital fundamental groups of isomorphic digital images are isomorphic as groups. Karaca and Vergili [12] also prove that isomorphic digital images have isomorphic homotopy groups in each dimension. Therefore they are invariants in digital topology and used in classifying the digital images.

Let $m$ be a positive integer. For a pointed digital image $\left(X, x_{0}\right)$, a $\kappa$-loop based at $x_{0}$ is a $(2, \kappa)$-continuous function $f:[0, m]_{\mathbb{Z}} \rightarrow X$ such that $f(0)=x_{0}=f(m)$ (see [13]).

Definition 3.1. [3] Let $f, g:[0, m]_{\mathbb{Z}} \rightarrow X$ be $\kappa$-loops such that

$$
f(0)=f(m)=g(0)=g(m)=x_{0} \in X .
$$

If

$$
H:[0, m]_{\mathbb{Z}} \times[0, M]_{\mathbb{Z}} \rightarrow X
$$

is a digital homotopy such that $H(0, t)=H(m, t)=x_{0}$ for all $t \in[0, M]_{Z}$, then we say $H$ holds the endpoints fixed.
Khalimsky [13] defines an operation between $\kappa$-loops with same base points as follows. Let $f:\left[0, m_{1}\right]_{\mathbb{Z}} \rightarrow X$, $g:\left[0, m_{2}\right]_{\mathbb{Z}} \rightarrow X$ be two $\kappa$-loops at based $x_{0}$. Then the map $f * g:\left[0, m_{1}+m_{2}\right]_{\mathbb{Z}} \rightarrow X$ defined by

$$
(f * g)(t)= \begin{cases}f(t), & 0 \leq t \leq m_{1} \\ g\left(t-m_{1}\right), & m_{1} \leq t \leq m_{1}+m_{2}\end{cases}
$$

is also a $\kappa$-loop based at $x_{0}$.

The number $m$ depends on the loop. Different loops have digital interval domains with different cardinality. The notion of trivial extension given in [2] allows two different loops to have same domains. So, they can be remain in the same digital homotopy class.

The homotopy holding the endpoints fixed, is an equivalence relation on the set of all $\kappa$-loops with same base point in $X$. The loops $f, g$ belong the same loop class $[f][3]$ if they have trivial extensions that can be deformed to each other by a homotopy that holds the endpoints fixed. The set of all equivalence classes is denoted by $\pi_{1}^{\kappa}\left(X, x_{0}\right)$.

The following proposition shows that the operation ${ }^{*}$, is well defined on equivalence classes.

Proposition 3.2. [2,13] Let $f_{1}, f_{2}, g_{1}, g_{2}$ be digital $\kappa$-loops with base point $x_{0}$ in a digital image $X$. Suppose $f_{2} \in\left[f_{1}\right]$ and $g_{2} \in\left[g_{1}\right]$. Then $f_{2} * g_{2} \in\left[f_{1} * g_{1}\right]$.

Theorem 3.3. [2] $\pi_{1}^{\kappa}\left(X, x_{0}\right)$ is a group under the product operation '.' defined as $[f] .[g]=[f * g]$.

Proposition 3.4. [3] If $\left(X, x_{0}\right)$ is a pointed $\kappa$-contractible digital image, then $\pi_{1}^{\kappa}\left(X, x_{0}\right)$ is a trivial group.

The $n$-boundary of $[0, m]_{\mathbb{Z}}^{n}$, denoted by $\partial[0, m]_{\mathbb{Z}}$, is defined as follows:

$\partial[0, m]_{\mathbb{Z}}^{n}=\left\{\left(t_{1}, \ldots, t_{n}\right): \exists i \in\{1,2, . ., m\} t_{i}=0\right.$ or $\left.t_{i}=m\right\}$.

Let $\left(X, x_{0}\right)$ be a pointed digital image with $\kappa$-adjacency relation. Let $S_{n}^{\kappa}\left(X, x_{0}\right)$ [12] be the set of all $(2 n, \kappa)$-continuous maps of the form

$$
f:\left([0, m]_{\mathbb{Z}}^{n}, \partial[0, m]_{\mathbb{Z}}^{n}\right) \longrightarrow\left(X, x_{0}\right) .
$$

Karaca and Vergili [12] show that homotopy relation relative to $\partial[0, m]_{\mathbb{Z}}^{n}$ is an equivalence relation on $S_{n}^{\kappa}\left(X, x_{0}\right)$. The set of all equivalence classes denoted by $\pi_{n}^{\kappa}(X, p)$ and the equivalence class of $f \in S_{n}^{\kappa}(X, p)$ is denoted by $[f]$.

Definition 3.5. [12] Let $\left(X, x_{0}\right)$ be a pointed digital image with $\kappa$ adjacency and

$$
f:\left([0, m]_{\mathbb{Z}}^{n}, \partial[0, m]_{\mathbb{Z}}^{n}\right) \longrightarrow(X, p)
$$


be $(2 n, \kappa)$-continuous map. If there is a positive integer integer $m_{1} \geq m$, and a map

$$
f^{\prime}:\left(\left[0, m_{1}\right]_{\mathbb{Z}}^{n}, \partial\left[0, m_{1}\right]_{\mathbb{Z}}^{n}\right) \longrightarrow(X, p)
$$

defined as

$$
f^{\prime}\left(t_{1}, \ldots, t_{n}\right)= \begin{cases}f(t), & 0 \leq \forall t_{i} \leq m, \quad i=1,2, \ldots, n \\ x_{0}, & \text { otherwise. }\end{cases}
$$

for $t=\left(t_{1}, \ldots, t_{n}\right) \in\left[0, m_{1}\right]_{\mathbb{Z}}^{n}$ then $f^{\prime}$ is called the trivial extension of $f$.

Digital $(2 n, \kappa)$-continuous maps $f$ and $g$ in $S_{n}^{\kappa}\left(X, x_{0}\right)$ are in the same equivalence class in $\pi_{n}^{\kappa}\left(X, x_{0}\right)$ if there are trivial extensions $f^{\prime}$ and $g^{\prime}$ of $f$ and $g$, respectively, and a relative digital homotopy between $f^{\prime}$ and $g^{\prime}$.

Definition 3.6. [12] Let $\left(X, x_{0}\right)$ be a pointed digital image with $\kappa$-adjacency relation. Let

$$
f:\left(\left[0, m_{1}\right]_{\mathbb{Z}}^{n}, \partial\left[0, m_{1}\right]_{\mathbb{Z}}^{n}\right) \longrightarrow\left(X, x_{0}\right)
$$

and

$$
g:\left(\left[0, m_{2}\right]_{\mathbb{Z}}^{n}, \partial\left[0, m_{2}\right]_{\mathbb{Z}}^{n}\right) \longrightarrow\left(X, x_{0}\right)
$$

be $(2 n, \kappa)$-continuous maps. The 'product' of $f$ and $g$, written $f \star g$, is defined as

$$
\begin{gathered}
(f \star g):\left(\left[0, m_{1}+m_{2}\right]_{\mathbb{Z}}^{n}, \partial\left[0, m_{1}+m_{2}\right]_{\mathbb{Z}}^{n}\right) \longrightarrow\left(X, x_{0}\right) \\
(f \star g)(t)= \begin{cases}f\left(t_{1}, \ldots, t_{n}\right), & t_{1} \in\left[0, m_{1}\right]_{\mathbb{Z}} \\
g\left(t_{1}-m_{1}, \ldots, t_{n}\right), & \text { and for } \left.j \neq 1, t_{j} \leq m_{1}, m_{1}+m_{2}\right]_{\mathbb{Z}} \\
& \text { and for } j \neq 1, t_{j} \leq m_{2} ; \\
x_{0}, & \text { otherwise. }\end{cases}
\end{gathered}
$$

Karaca and Vergili [12] show that the operation

$$
[f] \star[g]=[f \star g]
$$

is well-defined on $\pi_{n}^{\kappa}\left(X, x_{0}\right)$ and the set $\pi_{n}^{\kappa}\left(X, x_{0}\right)$ has a group structure via ' $\star$ ' operation. This group is called a digital $n$-th homotopy group of a pointed digital image $\left(X, x_{0}\right)$. Actually this construction coincides with the fundamental group construction which is given by Boxer [2], when $n=1$.

Let $\left(X, x_{0}\right),\left(Y, y_{0}\right)$ be two digital images with $\kappa_{1}, \kappa_{2}$ adjacency relations respectively and the digital map $\varphi:(X, p) \longrightarrow(Y, q)$ be a $\left(\kappa_{1}, \kappa_{2}\right)$-continuous. Homomorphism induced by $\varphi$ [12] is defined as follows:

$$
\varphi_{*}: \pi_{n}^{\kappa_{1}}\left(X, x_{0}\right) \rightarrow \pi_{n}^{\kappa_{2}}\left(Y, y_{0}\right), \quad[f] \mapsto \varphi_{*}([f])=[\varphi \circ f] .
$$

Theorem 3.7. [12] The digital homotopy group construction induces a covariant functor from the category of pointed digital images and pointed digitally continuous functions to the category of groups and homomorphisms.

Corollary 3.8. [12] Let $\left(X, x_{0}\right)$ be pointed $\kappa$-contractible. Then $\pi_{n}^{\kappa}\left(X, x_{0}\right)$ is trivial for all positive integer $n$.

\section{Computing Higher Digital Homotopy Groups}

In this section, we introduce a method for computing homotopy groups of digital images via covering spaces. We prove that a radius 2 local isomorphism induces an isomorphism between higher dimensional digital homotopy groups.

Definition 4.1. ([9]) Let $\left(E, \kappa_{0}\right)$ and $\left(B, \kappa_{1}\right)$ be digital images and $p: E \rightarrow B$ be a $\left(\kappa_{0}, \kappa_{1}\right)$-continuous surjection. Suppose for any $b \in B$ there exists $\varepsilon \in \mathbb{N}$ such that

(DC 1) For some $\delta \in \mathbb{N}$ and some index set $M$, $p^{-1}\left(N_{\kappa_{1}}(b, \varepsilon)\right)=\bigcup_{i \in M} N_{\kappa_{0}}\left(e_{i}, \delta\right)$ with $e_{i} \in p^{-1}(b)$;

(DC 2) if $i, j \in M$ and $i \neq j$, then

$$
N_{\kappa_{0}}\left(e_{i}, \delta\right) \cap N_{\kappa_{0}}\left(e_{j}, \delta\right)=\emptyset
$$

(DC 3) the restriction map

$$
\left.p\right|_{N_{\kappa_{0}}\left(e_{i}, \delta\right)}: N_{\kappa_{0}}\left(e_{i}, \delta\right) \rightarrow N_{\kappa_{1}}(b, \varepsilon)
$$

is a $\left(\kappa_{0}, \kappa_{1}\right)$-isomorphism for all $i \in M$.

Then the map $p$ is called a $\left(\kappa_{0}, \kappa_{1}\right)$-covering map and $(E, p, B)$ is a $\left(\kappa_{0}, \kappa_{1}\right)$-covering.

Boxer [4] shows that the following proposition is equivalent to the definition of digital covering maps.

Proposition 4.2. ([4]) Let $\left(E, \kappa_{0}\right)$ and $\left(B, \kappa_{1}\right)$ be digital images and $p: E \rightarrow B$ be a $\left(\kappa_{0}, \kappa_{1}\right)$-continuous surjection. Then the map $p$ is a $\left(\kappa_{0}, \kappa_{1}\right)$-covering map if and only if for each $b \in B$ there exist an index set $M$ such that

with $e_{i} \in p^{-1}(b)$;

$$
(\mathrm{C} 1) p^{-1}\left(N_{\kappa_{1}}(b, 1)\right)=\bigcup_{i \in M} N_{\kappa_{0}}\left(e_{i}, 1\right)
$$

(C 2) if $i, j \in M$ and $i \neq j$, then

$$
N_{\kappa_{0}}\left(e_{i}, 1\right) \cap N_{\kappa_{0}}\left(e_{j}, 1\right)=\emptyset ;
$$

(C 3) the restriction map

$$
\left.p\right|_{N_{\kappa_{0}}\left(e_{i}, 1\right)}: N_{\kappa_{0}}\left(e_{i}, 1\right) \rightarrow N_{\kappa_{1}}(b, 1)
$$


is a $\left(\kappa_{0}, \kappa_{1}\right)$-isomorphism for all $i \in M$.

Definition 4.3. ([10]) For $n \in \mathbb{N}$, a $\left(\kappa_{0}, \kappa_{1}\right)$-covering $(E, p, B)$ is a radius $n$ local isomorphism if the restriction map

$$
\left.p\right|_{N_{\kappa_{0}}\left(e_{i}, n\right)}: N_{\kappa_{0}}\left(e_{i}, n\right) \rightarrow N_{\kappa_{n}}(b, n)
$$

is a $\left(\kappa_{0}, \kappa_{1}\right)$-isomorphism for all $i \in M$.

By Proposition 4.2 (C 3), we know that every covering map is a radius 1 local isomorphism. A digital simple closed $\kappa$-curve is a digital image $S=\left\{c_{i}\right\}_{i=0}^{m-1}$ such that $s_{i}$ and $s_{j}$ are $\kappa$-adjacent if and only if either $j \equiv i+1$ $\bmod m$ or $j \equiv i-1 \bmod m . p: \mathbb{Z} \rightarrow S, \quad p(z)=c_{z \bmod m}$ is a $(2, \kappa)$-covering map [9]. Hence it is a radius 1 local isomorphism. However, if $S=M S C_{8}^{\prime}$ which is isomorphic to digital image

$$
\left\{c_{0}=(1,0), c_{1}=(0,1), c_{2}=(-1,0), c_{3}=(0,-1)\right\},
$$

then $p$ is not radius 2 local isomorphism [4]. That is a covering map doesn't need to be a radius 2 local isomorphism. We give another example in respect to this as below.

Example 4.4. Boxer [5] defines a digital $n$-sphere as

$$
S_{n}=[-1,1]_{\mathbb{Z}}^{n+1}-\left\{\mathbf{0}_{n+1}\right\}
$$

where $\mathbf{0}_{n}$ represents the origin of $\mathbb{Z}^{n}$. We get

$$
\begin{aligned}
S_{2}=\left\{c_{0}\right. & =(-1,-1,-1), c_{1}=(-1,0,-1), c_{2}=(-1,1,-1) \\
& c_{3}=(0,1,-1), c_{4}=(0,0,-1), c_{5}=(0,-1,-1), \\
& c_{6}=(1,-1,-1), c_{7}=(1,0,-1), c_{8}=(1,1,-1), \\
& c_{9}=(1,1,0), c_{10}=(1,0,0), c_{11}=(1,-1,0), \\
& c_{12}=(0,-1,0), c_{13}=(0,1,0), c_{14}=(-1,1,0), \\
& c_{15}=(-1,0,0), c_{16}=(-1,-1,0), c_{17}=(-1,-1,1), \\
& c_{18}=(-1,0,1), c_{19}=(-1,1,1), c_{20}=(0,1,1), \\
& c_{21}=(0,0,1), c_{22}=(0,-1,1), c_{23}=(1,-1,1), \\
& \left.c_{24}=(1,0,1), c_{25}=(1,1,1)\right\}
\end{aligned}
$$

Let $q: S_{2} \rightarrow S_{2} / x \sim-x$ be the quotient map where $-x$ is the antipodal point of $x$ in $S_{2}$. The quotient space is as follows:

\section{$\left\{\left[c_{0}\right],\left[c_{1}\right],\left[c_{2}\right],\left[c_{3}\right],\left[c_{4}\right],\left[c_{5}\right],\left[c_{6}\right],\left[c_{7}\right],\left[c_{8}\right],\left[c_{9}\right],\left[c_{13}\right],\left[c_{14}\right],\left[c_{15}\right]\right\}$}

It is called digital projective plane and denoted by $P^{2}$. (See Figure 1)

Note that $\left(P^{2}, x\right)$ is pointed 6-contractible for all $x \in P^{2}$. For example, the contracting 6-homotopy $H: P^{2} \times[0,5]_{\mathbb{Z}} \longrightarrow P^{2}$ of the pointed digital image $\left(P^{2},\left[c_{0}\right]\right)$ can be defined as follows:
For all $i \in\{0,1,2,3,4,5,6,7,8,9,13,14,15\}$

$H\left(\left[c_{i}\right], 0\right)=\left[c_{i}\right]$;

for all $i \in\{0,1,2,3,4,5,6,7,8\} \quad H\left(\left[c_{i}\right], 1\right)=\left[c_{i}\right]$,

$H\left(\left[c_{9}\right], 1\right)=\left[c_{8}\right]$,

$H\left(\left[c_{13}\right], 1\right)=\left[c_{3}\right]$,

$H\left(\left[c_{14}\right], 1\right)=\left[c_{2}\right]$,

$H\left(\left[c_{15}\right], 1\right)=\left[c_{1}\right]$;

for all $i \in\{0,1,4,5,6,7\} \quad H\left(\left[c_{i}\right], 2\right)=\left[c_{i}\right]$, for all $i \in\{2,14,15\} \quad H\left(\left[c_{i}\right], 2\right)=\left[c_{1}\right]$, for all $i \in\{3,13\} \quad H\left(\left[c_{i}\right], 2\right)=\left[c_{4}\right]$, for all $i \in\{8,9\} \quad H\left(\left[c_{i}\right], 2\right)=\left[c_{7}\right]$;

for all $i \in\{1,2,14,15\} \quad H\left(\left[c_{i}\right], 3\right)=\left[c_{0}\right]$, for all $i \in\{3,4,13\} \quad H\left(\left[c_{i}\right], 3\right)=\left[c_{5}\right]$, for all $i \in\{7,8,9\} \quad H\left(\left[c_{i}\right], 3\right)=\left[c_{6}\right]$, for all $i \in\{0,5,6\} \quad H\left(\left[c_{i}\right], 3\right)=\left[c_{i}\right]$;

for all $i \in\{1,2,14,15\} \quad H\left(\left[c_{i}\right], 4\right)=\left[c_{0}\right]$, for all $i \in\{3,4,6,7,8,9,13\} \quad H\left(\left[c_{i}\right], 4\right)=\left[c_{5}\right]$ for all $i \in\{0,5\} \quad H\left(\left[c_{i}\right], 4\right)=\left[c_{i}\right]$;

for all $i \in\{0,1,2,3,4,5,6,7,8,9,13,14,15\}$ $H\left(\left[c_{i}\right], 5\right)=\left[c_{0}\right]$.

The quotient map $q$ is a $(6,6)$-continuous surjection and it can be easily seen that $q$ satisfies the conditions of Proposition 4.2. Hence $q$ is a $(6,6)$-covering map. But it isn't a radius 2 local isomorphism. For $\left[c_{1}\right] \in P^{2}$, we obtain

$$
N_{6}\left(\left[c_{1}\right], 2\right)=\left\{\left[c_{0}\right],\left[c_{1}\right],\left[c_{3}\right],\left[c_{4}\right],\left[c_{5}\right],\left[c_{7}\right],\left[c_{14}\right],\left[c_{15}\right]\right\}
$$

and

$$
N_{6}\left(c_{1}, 2\right)=\left\{c_{0}, c_{1}, c_{2}, c_{3}, c_{4}, c_{5}, c_{7}, c_{14}, c_{15}, c_{16}, c_{18}\right\} .
$$

Since $N_{6}\left(c_{1}, 2\right)$ and $N_{6}\left(\left[c_{1}\right], 2\right)$ don't have the same cardinality,

$$
\left.q\right|_{N_{6}\left(c_{1}, 2\right)}: N_{6}\left(c_{1}, 2\right) \rightarrow N_{6}\left(\left[c_{1}\right], 2\right)
$$

cannot be a $(6,6)$-isomorphism.

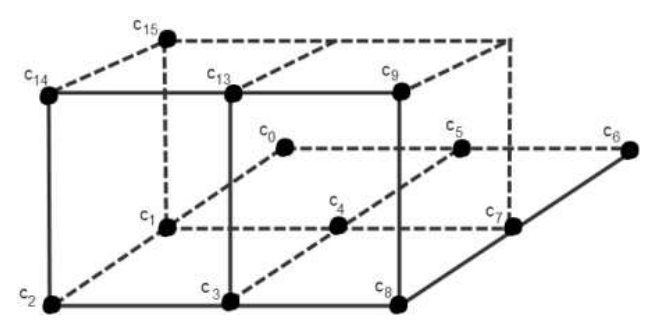

Fig. 1: Digital Projective Plane $P^{2}$

Let $\left(E, \kappa_{0}\right),\left(B, \kappa_{1}\right)\left(X, \kappa_{2}\right)$ be digital images and $p: E \rightarrow B$ be a $\left(\kappa_{0}, \kappa_{1}\right)$-covering map. Let $f: X \rightarrow B$ be 
$\left(\kappa_{2}, \kappa_{1}\right)$-continuous function. A digital lifting [10] of $f$ with respect to $p$ is a $\left(\kappa_{2}, \kappa_{0}\right)$-continuous function $\tilde{f}: X \rightarrow E$ such that $p \circ \tilde{f}=f$.

A digital continuous function doesn't need to have liftings. But we can determine whether a function has a lifting or not with the help of the following theorem.

Theorem 4.5. [4] Let $\left(E, \kappa_{0}\right),\left(B, \kappa_{1}\right)$ be pointed digital images with $e_{0} \in E, b_{0} \in B$ and let $p:\left(E, e_{0}\right) \rightarrow\left(B, b_{0}\right)$ be a pointed $\left(\kappa_{0}, \kappa_{1}\right)$-covering map. Let $X$ be a $\kappa_{2}$-connected digital image, $x_{0} \in X$. Let $\phi:\left(X, x_{0}\right) \rightarrow\left(B, b_{0}\right)$ be a pointed $\left(\kappa_{2}, \kappa_{1}\right)$-continuous map. Consider the following statements:

(a) There exists a lifting $\tilde{\phi}:\left(X, x_{0}\right) \rightarrow\left(E, e_{0}\right)$ of $\phi$ with respect to $p$.

(b) $\phi_{*}\left(\pi_{1}^{\kappa_{2}}\left(X, x_{0}\right)\right) \subseteq p_{*}\left(\pi_{1}^{\kappa_{0}}\left(E, e_{0}\right)\right)$.

Then $(a)$ implies $(b)$. Further, if $p$ is a radius 2 local isomorphism, then $(b)$ implies $(a)$.

Han [9] shows that for a pointed $\left(\kappa_{0}, \kappa_{1}\right)$-covering map $p:\left(E, e_{0}\right) \longrightarrow\left(B, b_{0}\right)$, any $\kappa_{1}$-path $f:[0, m]_{\mathbb{Z}} \longrightarrow B$ beginning at $b_{0}$ has a unique digital lifting to a $\kappa_{0}$-path $\tilde{f}$ in $E$ beginning at $e_{0}$. We give a generalization of Han's result.

A pointed digital image $\left(X, x_{0}\right)$ is said to be simply $\kappa$-connected [9] if $\pi_{1}^{\kappa}\left(X, x_{0}\right)$ is a trivial group.

Proposition 4.6. Let $\left(E, \kappa_{0}\right)$ be a digital image and $e_{0} \in E$. Let $\left(B, \kappa_{1}\right)$ be a digital image and $b_{0} \in B$. Let $p:\left(E, e_{0}\right) \rightarrow\left(B, b_{0}\right)$ be a pointed $\left(\kappa_{0}, \kappa_{1}\right)$-covering map which is a radius 2 local isomorphism. Suppose that $\left(X, x_{0}\right)$ is a simply $\kappa_{2}$-connected digital image and $A$ is $\kappa_{2}$-connected nonempty subset of $X$ such that $x_{0} \in A$. Then any $\left(\kappa_{2}, \kappa_{1}\right)$-continuous map $f:(X, A) \rightarrow\left(B, b_{0}\right)$ has a lifting to a $\left(\kappa_{2}, \kappa_{0}\right)$-continuous map $\tilde{f}:(X, A) \rightarrow\left(E, e_{0}\right)$.

Proof. Let $x_{0} \in A$. We can take $f:\left(X, x_{0}\right) \rightarrow\left(B, b_{0}\right)$ as a $\left(\kappa_{2}, \kappa_{1}\right)$-continuous map of pointed digital images. There is an induced homomorphism

$$
f_{*}: \pi_{1}^{\kappa_{2}}\left(X, x_{0}\right) \rightarrow \pi_{1}^{\kappa_{1}}\left(B, b_{0}\right)
$$

between digital fundamental groups. Since $X$ is a simply $\kappa_{2}$-connected digital image, it follows that

$$
f_{*}\left(\pi_{1}^{\kappa_{2}}\left(X, x_{0}\right)\right) \subset p_{*}\left(\pi_{1}^{\kappa_{0}}\left(E, e_{0}\right)\right) .
$$

From the previous theorem, there is a lifting $\tilde{f}:\left(X, x_{0}\right) \rightarrow\left(E, e_{0}\right)$ of $f$ such that $p \circ \tilde{f}=f$.

Let $x_{1}$ be $\kappa_{2}$-adjacent to $x_{0}$ in $A$. Since $\tilde{f}$ is a $\left(\kappa_{2}, \kappa_{0}\right)$ continuous map, $\tilde{f}\left(x_{1}\right)$ must be in $N_{\kappa_{0}}\left(\tilde{f}\left(x_{0}\right), 1\right)$. Since $p$ is a $\left(\kappa_{0}, \kappa_{1}\right)$-covering map,

$$
\left.p\right|_{N_{\kappa_{0}}\left(\tilde{f}\left(x_{0}\right), 1\right)}: N_{\kappa_{0}}\left(\tilde{f}\left(x_{0}\right), 1\right) \rightarrow N_{\kappa_{1}}\left(b_{0}, 1\right)
$$

is a $\left(\kappa_{0}, \kappa_{1}\right)$-isomorphism. We get

$$
\begin{aligned}
\left.p\right|_{N_{\kappa_{0}}\left(\tilde{f}\left(x_{0}\right), 1\right)} \circ \tilde{f}\left(x_{0}\right) & =f\left(x_{0}\right)=b_{0}=f\left(x_{1}\right) \\
& =\left.p\right|_{N_{\kappa_{0}}\left(\tilde{f}\left(x_{0}\right), 1\right)} \circ \tilde{f}\left(x_{1}\right) .
\end{aligned}
$$

Hence we have $\tilde{f}\left(x_{1}\right)=\tilde{f}\left(x_{0}\right)=e_{0}$. Since $A$ is a $\kappa_{2}$-connected digital image, we can iterate this argument for any point of $A$. This shows that $\tilde{f}(A)=e_{0}$. Hence we can consider the map $\tilde{f}$ as a map of digital pairs

$$
\widetilde{f}:(X, A) \longrightarrow\left(E, e_{0}\right) .
$$

Consequently, we get a lifting $\tilde{f}$ of $f:(X, A) \longrightarrow\left(B, b_{0}\right)$ with respect to $p$.

Lemma 4.7. Let $p:\left(E, e_{0}\right) \rightarrow\left(B, b_{0}\right)$ be a pointed $\left(\kappa_{0}, \kappa_{1}\right)$ covering map which is a radius 2 local isomorphism. Then any $\left(2 n, \kappa_{1}\right)$-continuous map

$$
f:\left([0, m]_{\mathbb{Z}}^{n}, \partial[0, m]_{\mathbb{Z}}^{n}\right) \rightarrow\left(B, b_{0}\right)
$$

has a unique lifting

$$
\tilde{f}:\left([0, m]_{\mathbb{Z}}^{n}, \partial[0, m]_{\mathbb{Z}}^{n}\right) \rightarrow\left(E, e_{0}\right)
$$

with respect to $p$.

Proof. The existence follows from Proposition 4.6, since $[0, m]_{\mathbb{Z}}^{n}$ is simply $2 n$-connected and its boundary is $2 n$-connected nonempty subset of $[0, m]_{\mathbb{Z}}^{n}$.

Let $\tilde{f}_{0}:\left([0, m]_{\mathbb{Z}}^{n}, \partial[0, m]_{\mathbb{Z}}^{n}\right) \rightarrow\left(E, e_{0}\right)$ be another lifting of $f$ with respect to $p$. It is known that $\tilde{f}(x)=\tilde{f}_{0}(x)$ for any $x$ in $\partial[0, m]_{\mathbb{Z}}^{n}$. Let $x_{1}$ and $x_{2}$ be any $2 n$-adjacent members of $[0, m]_{\mathbb{Z}}^{n}$ such that $\tilde{f}\left(x_{1}\right)=\tilde{f}_{0}\left(x_{1}\right)=b_{1}$. Since $X=\left\{x_{1}, x_{2}\right\}$ is a $2 n$-connected set, $\tilde{f}_{0}$ is $\left(2 n, \kappa_{0}\right)$-continuous map and $p$ is a $\left(\kappa_{0}, \kappa_{1}\right)$-covering map, we have

$$
\tilde{f}_{0}(X) \subset p^{-1}\left(N_{\kappa_{1}}\left(b_{1}, 1\right)\right)=\bigcup_{\alpha \in M} N_{\kappa_{0}}\left(e_{\alpha}, 1\right) .
$$

There is a unique index $\alpha_{0}$ such that $\tilde{f}_{0}(X) \subset N_{\kappa_{0}}\left(e_{\alpha_{0}}, 1\right)$ because the union is disjoint. Similarly, one can see that there is a unique index $\alpha_{1}$ such that $\tilde{f}(X) \subset N_{\kappa_{0}}\left(e_{\alpha_{1}}, 1\right)$. $\tilde{f}_{0}\left(x_{1}\right)=\tilde{f}\left(x_{1}\right)$ implies that $\alpha_{0}=\alpha_{1}$.

$$
\left.p\right|_{N_{\kappa_{0}}\left(e_{\alpha_{0}}, 1\right)}: N_{\kappa_{0}}\left(e_{\alpha_{0}}, 1\right) \rightarrow N_{\kappa_{1}}\left(b_{1}, 1\right)
$$

is an $\left(\kappa_{0}, \kappa_{1}\right)$-isomorphism and

$$
p \circ \tilde{f}\left(x_{2}\right)=f\left(x_{2}\right)=p \circ \tilde{f}_{0}\left(x_{2}\right)
$$

for $x_{2} \in N_{\kappa_{0}}\left(e_{\alpha_{0}}, 1\right)$. This shows that $\tilde{f}\left(x_{2}\right)=\tilde{f}_{0}\left(x_{2}\right)$. Since $[0, m]_{\mathbb{Z}}^{n}$ is $2 n$-connected, this shows how we 
propagate our knowledge that $\tilde{f}(x)=\tilde{f}_{0}(x)$ from $x \in \partial[0, m]_{\mathbb{Z}}^{n}$ to all $x \in[0, m]_{\mathbb{Z}}^{n}$. So $\tilde{f}=\tilde{f}_{0}$, that is, the lifting is unique.

The following proposition is a general version of the digital homotopy lifting theorem given by Han [10].

Proposition 4.8. Let $\left(E, \kappa_{0}\right)$ be a digital image and $e_{0} \in E$. Let $\left(B, \kappa_{1}\right)$ be a digital image and $b_{0} \in B$. Suppose $p:\left(E, e_{0}\right) \rightarrow\left(B, b_{0}\right)$ is a $\left(\kappa_{0}, \kappa_{1}\right)$-covering map which is a radius 2 local isomorphism. For the two digital $\left(2 n, \kappa_{0}\right)$ continuous maps

$$
f_{0}, f_{1}:\left([0, m]_{\mathbb{Z}}^{n}, \partial[0, m]_{\mathbb{Z}}^{n}\right) \rightarrow\left(E, e_{0}\right),
$$

if there is a digital homotopy relative to $\partial[0, m]_{\mathbb{Z}}^{n}$ from $p \circ f_{0}$ to $p \circ f_{1}$, then there is a digital homotopy relative to $\partial[0, m]_{\mathbb{Z}}^{n}$ from $f_{0}$ to $f_{1}$.

Proof. Let $H:[0, m]_{\mathbb{Z}}^{n} \times[0, k]_{\mathbb{Z}} \rightarrow B$ be a homotopy relative to $\partial[0, m]_{\mathbb{Z}}^{n}$ from $p \circ f_{0}$ to $p \circ f_{1}$. A homotopy between $f_{0}$ and $f_{1}$ can be defined with a similar method in the proof of Lemma 4.7. Define

$$
\widetilde{H}:[0, m]_{\mathbb{Z}}^{n} \times[0, k]_{\mathbb{Z}} \rightarrow E
$$

starting on the set

$$
R=\left(\partial[0, m]_{\mathbb{Z}}^{n} \times[0, k]_{\mathbb{Z}}\right) \cup\left([0, m]_{\mathbb{Z}}^{n} \times\{0\}\right)
$$

as

$$
\begin{gathered}
\widetilde{H}(s, t)=e_{0}, \text { for } s \in \partial[0, m]_{\mathbb{Z}}^{n}, t \in[0, k]_{\mathbb{Z}} \\
\widetilde{H}(s, 0)=f_{0}(s), \text { for } s \in[0, m]_{\mathbb{Z}}^{n} .
\end{gathered}
$$

Let $M=\left\{s_{1}, s_{2}\right\}$ be arbitrary $2 n$-adjacent points of $[0, m]_{\mathbb{Z}}^{n}$ and $N=[j-1, j]_{\mathbb{Z}}$ for any $j \in[1, k]_{\mathbb{Z}}$. Assume that $\widetilde{H}\left(s_{1}, j\right)=b$ and $p \circ \widetilde{H}=H$ at $\left(s_{1}, j\right)$. Then

$$
p \circ \widetilde{H}=H
$$

holds on $R \cup\left\{\left(s_{1}, j\right)\right\}$. Since $p$ is a radius 2-local isomorphism, there exist $N_{\kappa_{1}}(b, 2)$ and $\left\{N_{\kappa_{0}}\left(e_{\alpha}, 2\right): \alpha \in M\right\}$ such that

$$
p^{-1}\left(N_{\kappa_{1}}(b, 2)\right)=\bigcup_{\alpha \in M} N_{\kappa_{0}}\left(e_{\alpha}, 2\right)
$$

and

$$
H(M \times N) \subset N_{\kappa_{1}}(b, 2)
$$

We have

$$
\widetilde{H}\left(s_{1}, j\right) \in p^{-1}\left(N_{\kappa_{1}}(b, 2)\right)=\bigcup_{\alpha \in M} N_{\kappa_{0}}\left(e_{\alpha}, 2\right) .
$$

Thus there is a unique neighborhood $N_{\kappa_{0}}\left(e_{\alpha_{0}}, 2\right)$ such that $\widetilde{H}\left(s_{1}, j\right) \in N_{\kappa_{0}}\left(e_{\alpha_{0}}, 2\right)$.

$$
\left.p\right|_{N_{\kappa_{0}}\left(e_{\alpha_{0}}, 2\right)}: N_{\kappa_{0}}\left(e_{\alpha_{0}}, 2\right) \rightarrow N_{\kappa_{1}}(b, 2)
$$

is a $\left(\kappa_{0}, \kappa_{1}\right)$-isomorphism. We can extend $\widetilde{H}$ by defining

$$
\widetilde{H}(s, t)=\left(\left.p\right|_{N_{\kappa_{0}}\left(e_{\alpha_{0}}, 2\right)}\right)^{-1} \circ H(s, t)
$$

for all $(s, t) \in(M \times N) \backslash\left\{\left(s_{1}, j\right)\right\}$. Since $[0, m]_{\mathbb{Z}}^{n} \times[0, k]_{\mathbb{Z}}$ is $2(n+1)$-connected, we define $\widetilde{H}$ on all $(s, t) \in[0, m]_{\mathbb{Z}}^{n} \times[0, k]_{\mathbb{Z}}$.

From the definition of $\widetilde{H}$ on $R, \widetilde{H}(s, 0)=f_{0}(s)$ for all $s \in[0, m]_{\mathbb{Z}}^{n}$.

$$
\widetilde{H}_{k}:[0, m]_{\mathbb{Z}}^{n} \rightarrow E, \quad s \mapsto \widetilde{H}_{k}(s)=\widetilde{H}(s, k)
$$

is the lifting of $H_{k}=p \circ f_{1}$. By Lemma 4.7, $\widetilde{H}_{k}=f_{1}$, i.e., $\widetilde{H}(s, k)=f_{1}(s)$ for all $s \in[0, m]_{\mathbb{Z}}^{n}$. Thus $\widetilde{H}$ is a digital homotopy between $f_{0}$ and $f_{1}$. As $\widetilde{H}(s, t)=e_{0}$ for any $s \in \partial[0, m]_{\mathbb{Z}}^{n}$ and $t \in[0, k]_{\mathbb{Z}}, \widetilde{H}$ is a digital homotopy relative to $\partial[0, m]_{\mathbb{Z}}^{n}$.

The following result gives a method for computing homotopy groups of digital images in higher dimensions.

Theorem 4.9. Let $\left(E, \kappa_{0}\right)$ be a digital image and $e_{o} \in E$. Let $\left(B, \kappa_{1}\right)$ be a digital image and $b_{0} \in B$. Suppose $p:\left(E, e_{0}\right) \rightarrow\left(B, b_{0}\right)$ is a $\left(\kappa_{0}, \kappa_{1}\right)$-covering map which is a radius 2 local isomorphism. Then the induced homomorphism

$$
\begin{aligned}
p_{*}: \pi_{n}^{\kappa_{0}}\left(E, e_{0}\right) & \longrightarrow \pi_{n}^{\kappa_{1}}\left(B, b_{0}\right) \\
{[f] } & \longmapsto \pi_{*}([f])=[p \circ f]
\end{aligned}
$$

is a group isomorphism for $n>1$.

Proof. Karaca and Vergili [12] show that $p_{*}$ is a group homomorphism from $\pi_{n}^{\kappa_{0}}\left(E, e_{0}\right)$ to $\pi_{n}^{\kappa_{1}}\left(B, b_{0}\right)$. By Proposition 4.6 and Proposition 4.8, the induced homomorphism is bijective. As a result, it is a group isomorphism.

The results of Boxer [6, Theorem 3.1] can be generalized to the higher dimensional digital homotopy groups of the unbounded digital images. Let $(X, \kappa)$ be a digital image such that $X=\cup_{j=1}^{\infty} X_{j}$ and for all $j$

$$
X_{j} \subset X_{j+1} \text { and }
$$$$
X_{j} \text { is bounded. }
$$

If the induced homomorphism

$$
\left(i_{j}\right)_{*}: \pi_{n}^{\kappa}\left(X_{j}\right) \longrightarrow \pi_{n}^{\kappa}\left(X_{j+1}\right)
$$

of the inclusion maps

$$
i_{j}: X_{j} \hookrightarrow X_{j+1}
$$

are isomorphisms for all $j$ and for all $n \geq 1$, then the inclusion map

$$
i_{X}: X_{1} \hookrightarrow X
$$


induces an isomorphism

$$
\left(i_{X}\right)_{*}: \pi_{n}^{\kappa}\left(X_{1}\right) \longrightarrow \pi_{n}^{\kappa}(X)
$$

for all $n \geq 1$.

This helps us to compute the digital homotopy groups of $(\mathbb{Z}, 0)$. To see this if we take $X_{j}=[-j, j]$ for $j=1,2, \ldots$. Since $X_{j}$ 's are contractible, $\pi_{n}^{2}\left(X_{j}\right)$ is trivial for all $n \geq 1$ and $\left(i_{j}\right)_{*}$ are isomorphisms for all $j=1,2, \ldots$. This leads us that $\pi_{n}^{2}(\mathbb{Z}, 0)=\{0\}$.

Example 4.10. Boxer [5] defines the digital 1-sphere as

$$
S_{1}=\left([-1,1]_{\mathbb{Z}} \times[-1,1]_{\mathbb{Z}}\right)-\{(0,0)\}
$$

(See Figure 2).

$$
p:(\mathbb{Z}, 0) \rightarrow\left(S_{1}, c_{0}\right), \quad t \mapsto p(t)=c_{t}(\bmod 8)
$$

is a $(2,4)$-covering map which is a radius 2 local isomorphism. So it induces a $(2,4)$-isomorphism

$$
p_{*}: \pi_{n}^{2}(\mathbb{Z}, 0) \longrightarrow \pi_{n}^{4}\left(S_{1}, c_{0}\right)
$$

for $n>1$. As we mention before, $\pi_{n}^{2}(\mathbb{Z}, 0)$ is trivial. Therefore we obtain $\pi_{n}^{4}\left(S_{1}, c_{0}\right) \cong\{0\}$.

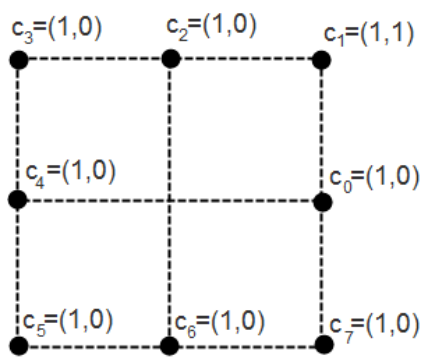

Fig. 2: Digital 1-Sphere $S_{1}$

Example 4.11. As the quotient map $q: S_{2} \rightarrow P^{2}$ is not a radius 2 local isomorphism, we cannot use Theorem 4.9 to compute $\pi_{n}^{6}\left(P^{2},\left[c_{0}\right]\right)$. But we still say that $\pi_{n}^{6}\left(P^{2},\left[c_{0}\right]\right)$ is trivial for $n>1$ because $\left(P^{2},\left[c_{0}\right]\right)$ is a pointed 6-contractible digital image.

\section{Conclusion}

In this paper we explore the relation between a digital image and its covering space. We obtain an isomorphism between higher dimensional homotopy groups of them when the covering map is a radius 2 local isomorphism. This is an alternative approach to compute digital homotopy groups in higher dimensions. In the future, we propose to compute higher dimensional homotopy groups of some digital surfaces and construct a digital image which has nontrivial digital homotopy groups.

\section{References}

[1] L. Boxer, Digitally continuous functions, Pattern Recognition Letters, 15, 833-839 (1994).

[2] L. Boxer, A classical construction for the digital fundamental group, Journal of Mathematical Imaging and Vision, 10, 51-62 (1999).

[3] L. Boxer, Properties of digital homotopy, Journal of Mathematical Imaging and Vision, 22, 19-26 (2005).

[4] L. Boxer, Digital products, wedges, and covering spaces, Journal of Mathematical Imaging and Vision, 25, 159-171 (2006).

[5] L. Boxer, Homotopy properties of sphere-like digital images, Journal of Mathematical Imaging and Vision, 24, 167-175 (2006).

[6] L. Boxer, Fundamental groups of unbounded digital images, Journal of Mathematical Imaging and Vision, 27, 121-127 (2007).

[7] L. Boxer, I. Karaca, The classification of digital covering spaces, Journal of Mathematical Imaging and Vision, 32, 2329 (2008).

[8] L. Boxer, I. Karaca, Some Properties of Digital Covering Spaces, Journal of Mathematical Imaging and Vision, 37, 17-26 (2010).

[9] BOXER, L. and KARACA, I., Actions of automorphism groups in a digital covering space, Journal of Pure and Applied Mathematics: Advances and Applications, 8, 4159 (2012).

[10] S.-E. Han, Digital Coverings and Their Applications, Journal of Applied Mathematics and Computing, 18, 487495 (2005).

[11] S.-E. Han, Connected sum of a digital closed surfaces, Information Sciences, 176, 332-348 (2006).

[12] I. Karaca, and T. Vergili, Some properties of higher dimensional homotopy groups for digital images, (preprint, 2012).

[13] E.Khalimsky, Motion, deformation, and homotopy in finite spaces, Proceedings IEEE International Conference on Systems, Man, and Cybernetics, 227-234 (1987).

[14] T.Y. Kong, A Digital Fundamental Group, Computers and Graphics, 13, 159-166 (1989).

[15] T.Y. Kong and A. Rosenfeld, Digital Topology- A Brief Introduction and Bibliograraphy, Topological Algarithms for the Digital Image Processing, Elsevier Science, Amsterdam, (1996).

[16] A. Rosenfeld, Continuous functions on digital pictures, Pattern Recognition Letters, 4, 177-184 (1986). 


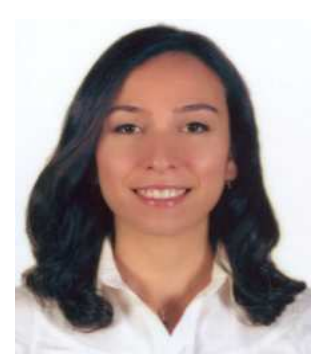

Elif Tugce Meric was born in Izmir, Turkey on December 1st, 1987. She received Bachelor's and Master's degrees in Mathematics from Ege University and started a $\mathrm{PhD}$ programme in the field of Algebra at Celal Bayar University. She is interested in Module Theory, Algebraic Topology and Digital Topology.

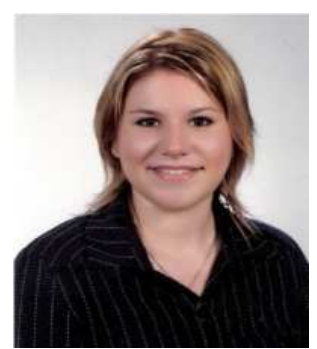

Tane Vergili was born in Izmir, Turkey on March 22nd, 1986. She received a Bachelor's degree in Mathematics from Ege University and started an integrated $\mathrm{PhD}$ programme in the field of Algebraic Topology at the same university. She is interested in Algebraic Topology and Digital Topology.

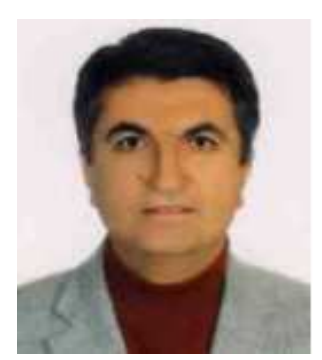

Ismet Karaca was born in Afyon, Turkey on January 5th, 1969. He received a Bachelor's degree in Mathematics from Anadolu University in Turkey, a Master's in Mathematics from the university of Miami, and a $\mathrm{PhD}$ in Mathematics from Lehigh University. $\mathrm{He}$ is a Professor of Mathematics at Ege University in Izmir, TURKEY. Dr. Karaca's research interests include Homotopy Theory, Steenrod Algebra, and Digital Topology. 